\title{
FROM THE DESK OF EDITOR in CHIEF
}

(J Bangladesh Coll Phys Surg 2018; 36: 43)

Greetings for Chrismas. Happy New Year. Hope this year will be blessed with memorable achievements.

We are trying hard to get international recognition of our journal. For this we need lot of quality articles regularly to publish four issues of the journal every year in time.

I invite our fellows specially juniors to contribute more in the field of research and submit their articles in the journal.
My earnest request to our respected reviewers to return the reviewed articles at their earliest convenient time.

Please stay with journal committee for ensuring better achievements.

Prof. Dr. Ferdousi Islam 